INTERNATIONAL DESIGN CONFERENCE - DESIGN 2018

https://doi.org/10.21278/idc.2018.0346

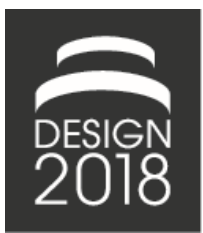

\title{
KNOWLEDGE-BASED SUPPORT DURING DESIGN OPTIMIZATION USING FLANGES AS AN EXAMPLE
}

\author{
A. Loibl, R. Andrae and P. Köhler
}

\begin{abstract}
In today's interdisciplinary environment, design engineers are facing increasing market demands for faster product cycle times. This implies that product-lifecycle processes need to be performed parallelly and faster. Repetitive processes should be questioned. To support the designer, processes can be automated by using clever linking software, Knowledge-Based Engineering (KBE), and a central knowledge base. This paper demonstrates an approach by giving examples of bolted and flange connections. The result supports the user, by providing simulation templates and optimization algorithms.
\end{abstract}

Keywords: knowledge based engineering (KBE), computer aided design (CAD), simulation based design

\section{Introduction}

In today's interdisciplinary environment, design engineers are facing increasing market demands for faster product cycle times, optimal material utilization, and shorter lead- and delivery times. This implies that product-lifecycle processes need to be performed in parallel and, above all, faster. Repetitive processes, time-consuming searches and documentations should be critically questioned, because they are costly - robbing valuable time from designers to focus on their actual tasks. To support the designers, such processes can be automated by using clever linking software, Knowledge-Based Engineering (KBE), and a central knowledge base.

Up to now, the conventional procedure for calculating and designing components has been to strictly comply with calculation specifications from rules, guidelines and standards. Although proven and legally secure, this design strategy, employed in many branches of industry, often leads to overdimensioning of the components and, thus, to unnecessary costs and wasted resources. One approach to address this problem is to switch from a classical partial model to central product models. This change avoids redundant data and, most of all, an independent existence of data. It also ensures that all models have access to the same dataset and always represent the current status. With regard to dimensioning by simulation, the iterative optimization of components is feasible and also ensures a continuous process chain.

An elementary prerequisite for such an approach is that it is based on a methodical procedure: It cannot be presupposed that a design engineer has the same knowledge as a calculation engineer. Thus, it is important to create a working environment that maximally supports a designer without overtaxing him or her. 


\section{State of the art}

The term "frontloading" describes the early use of optimized methods and IT-tools in product development. The application takes place during the product design phase during which $70-80 \%$ of product costs are committed, thereby offering the greatest potential for cost optimization. On the other hand, there is the greatest possible flexibility for corrections during this period. Whereas product information used to be stored in a single system, it is now referenced, even when stored in an external system. The designer, who is increasingly positioned at the center of an information system, is thus given the opportunity to use relevant information from internal and external sources (Szeghö and Bercesey, 2007).

Kesselmans describes frontloading as the attempt to test functions, operating behaviour, technological and other properties of a product to be developed as early on as possible in the development process with the help of digital models (Kesselmans, 2014). At the same time, he concludes that system support is still low during the conceptual and design phases. Regarding the early integration of numerical calculations, Hagenrainer notes that this seems to be sensible only by defining a support system for the designer (Hagenrainer, 2016).

The support of the design engineer in executing simulations and optimizations has always been an important research topic. Due to the increasing interlinking of the disciplines of design and calculation, the design engineer is expected to carry out advanced computations independently. Various guidelines provide the designer a methodical approach for applying computational methods in development and design processes (VDI, 2003).

It is demonstrated that safeguarding the multidisciplinary and manageability of advanced simulations through knowledge-based support systems is a widely used approach $[1,2,3]$. The methods for creating these are individual. In this context, one of the first approaches by Deng (2002), comprises a system for an integrated injection-moulded part design. By considering mainly the process chain CAD-CAE, they developed features oriented to injection-moulded parts and an interface between both systems. Moreover, Humpa and Köhler (2014) present a practical approach that includes a CAD-based geometry design approach for spiral-milled parts. Proposing KBE methods like User Defined Features (UDFs), combined with hybrid modelling techniques, these authors contend that rule-based model design improves the process (Humpa and Köhler, 2014): "By the use of methods and tools of KBE, both manufacturing process information and manufacturing knowledge can be integrated into the $3 \mathrm{D}$ product model."

A general procedure of creating a KBE system is contained in VDI 5610 Part 2 (VDI, 2017). As Kesselmans has already stated, the support of the designer can only take place if mechanisms are provided with which it is possible to process construction knowledge (Kesselmans, 2014). For this purpose, a platform must be made available in which this knowledge is stored: a knowledge base. Knowledge acquisition and the creation of the knowledge base are the basis of every KBE method. In view of demographic change, it is necessary to secure the knowledge that lies personalized with the designer und calculator for the further success of the company. It has to be extracted and structured. Techniques for acquiring of knowledge, both explicit and implicit, can be found in different sources such as (VDI, 2017).

Optimizing the CAD-CAE process chain allows numerical simulations to be executed more effectively. A first step is to use master models that include a geometric model for the CAD and an idealized model for the CAE. This ensures, for example, an automated dimensional reduction (Lee, 2005; Boussuge et al, 2014). Boussuge et al. (2014) proposes the use of an enriched digital mock-up with geometric interfaces between components and functional properties. His practical example comprises the use of bolted junctions in assembly context. The geometry of the CAD-models is separated and simplified in relevant subdomains that can be exported as a STEP file into CAE software.

During the design process, the designer can execute advanced simulations through a closer linkage of the CAD-CAE domains. Recent contributions in the area of simulations during the design process concern the development of knowledge-based FEA assistance systems (Kestel and Wartzack, 2016) as well as methods for a knowledge-based definition of (simulation-oriented) product models (Klemme, 2015). These help designers to perform and evaluate simulations. 
In many current contributions, the access to external data via the CAD-system is web-based. The papers of Danjou and Manoharan are noteworthy upon using the feature technology focused on variant constructions. Danjou et al. (2008) present the use of UDFs exemplified by an automated compressor configuration. The necessary parameters are stored in a database, and a UDF library is created for the individual UDFs. Depending on the requirements, a configurator places the UDFs in the CAD assembly. Moreover Manoharan et al. (2016) shows knowledge integration in the CAD-CAM process chain through the example of $\mathrm{NC}$ and additive manufacturing. Here, the author implements a web service for linking the CAD system with the respective databases (Figure 1).

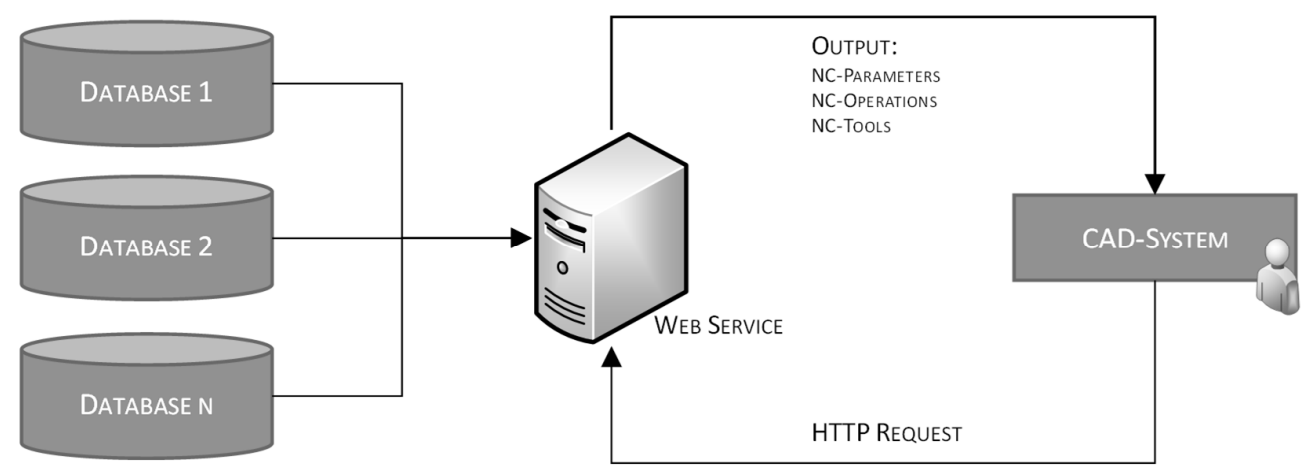

Figure 1. Linkage databases - web service - CAD-system (Manoharan et al., 2016)

According to Manoharan et al. (2016), one important advantage for using a web service is if changes are needed in the database structure. This ensures that only the web service or the database has to be adapted and not the expert system.

As this point, it can be concluded that the timeframe allocated to product development is becoming shorter and shorter. In addition, the amount of data is increasing rapidly. Therefore, the development of new methods and the optimization of existing procedures to support the designer are only logical. The early provision of information for the designer and simulation expert is still only covered to a certain extent by information technology solutions. It takes too much time to gather information, which is usually iterative, for executing the preprocessing. The iteration time depends on the user and the software systems applied. Here, it is important that methodical support in the sense of frontloading take place.

\section{Qualification of knowledge-based safeguards at the interface of CAD-CAE}

Often the development effort and benefits of knowledge-based systems are not correctly estimated. Yet this should be done in view of the general background of the construction type. The significance of the CAD model for the entire (computer-aided) product development has to be emphasized here, because a specific partial model can be derived and used in almost every phase. The following gives the construction types with their respective shares in the product development process (Roller, 1995):

- New construction $(12 \%)$

- Variant construction (33\%)

- Adaptation construction (55\%)

This classification shows that adaptation construction (55\%) has the greatest use. However, it needs to be pointed out here that adaptation constructions are usually a mix, consisting of a small fraction of new construction and a larger fraction of variant construction. In order to qualify a knowledge-based safeguarding of an adaptation design, it is necessary to analyse the degree to which the process chains of computer-integrated product development are linked in a CAD system; see Figure 2.

On both sides of Figure 2, an insufficient linking of individual partial models is exemplified. A subassembly of a support bracket is shown on the left-hand side. By default, only the geometry and the material are linked in the partial models whereas the respective views and parameters are usually not. 
This makes a semi-automated processing nearly impossible. In this example, this concerns geometry idealizations as well as boundary and load conditions.

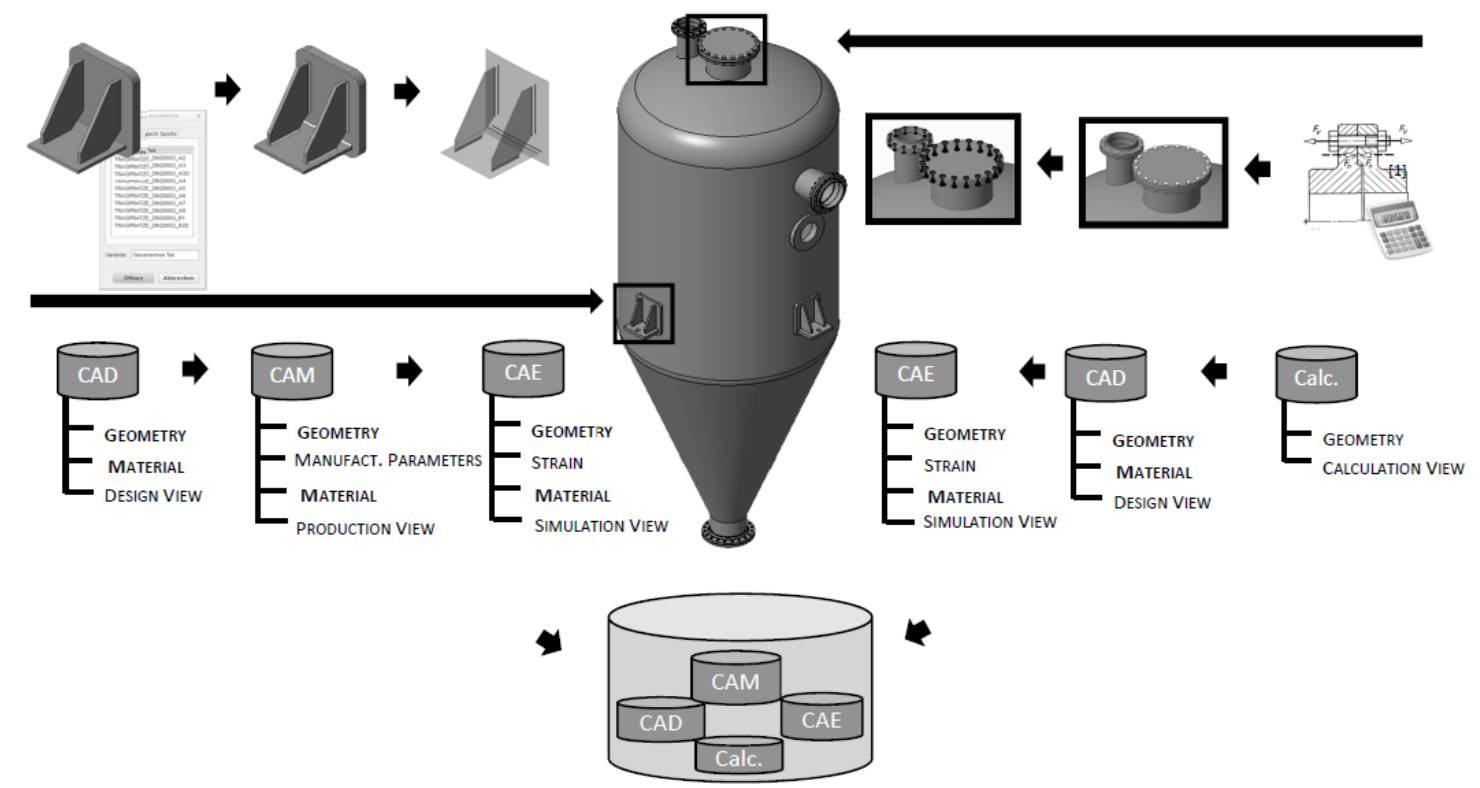

Figure 2. Linkage process chains

An unsatisfactory inclusion of analytical computations is shown in the blind flange connection on the right-hand side of the figure. A selected bolt connection is usually calculated analytically. The selection of the bolted connection also depends on the selected geometry of the flange connection. Similarly, in the case of an asymmetrical load, a numerical simulation has to check the gasket behavior. Yet the partial models are not sufficiently linked here.

The two selected process chains indicate missing links between partial models in the CAD system on the one hand, and insufficient methodological support of the designer on the other hand. Both examples show that new interdisciplinary features and component models are needed in the CAD system in which the individual partial models are predefined and linked. Moreover, a uniform database than can be equally accessed by all disciplines is required. Here, further approaches to address these issues require a cross-domain support system for the designer.

\section{Case study: Flange calculation}

Figure 3 features the general procedure for optimizing a component. Standard data, material data and characteristic values, numerical calculations and geometry parameters are stored in the central knowledge base. The user has the possibility to access a standard parts database while designing the CAD model. When switching to the CAE environment, the simulation model derives from the CAD model, which is already stored as a template and subordinate to the CAD model in which the meshing, bearing and contact conditions, prestressing forces and loads are stored. The CAE model also accesses the knowledge database and performs calculations based on stored templates, which themselves are based on standards or guidelines, that determine preload forces and design criteria. After the simulation is carried out, the results are compared with these standards and guidelines and, if necessary, optimized according to the optimization routine. Safety values and material limits are taken into account at all times. If a geometric adjustment is required, it is transferred to the CAD model and updated accordingly. For flange calculation, there are a number of standards and guidelines that have to be selected depending on the location of the flange and, thus, on the certification procedure. Widely used guidelines are DIN EN 1591-1 (DIN, 2013), VDI 2230 Part 2 (VDI, 2014), ASME Sec. VIII (ASME, 2013) and the AD2000 B7/B8 (DIN 2016, 2017) regulations. Even though this current paper considers only DIN EN 1591-1 (DIN, 2013), the developed approach ensures transferability to other regulations. 


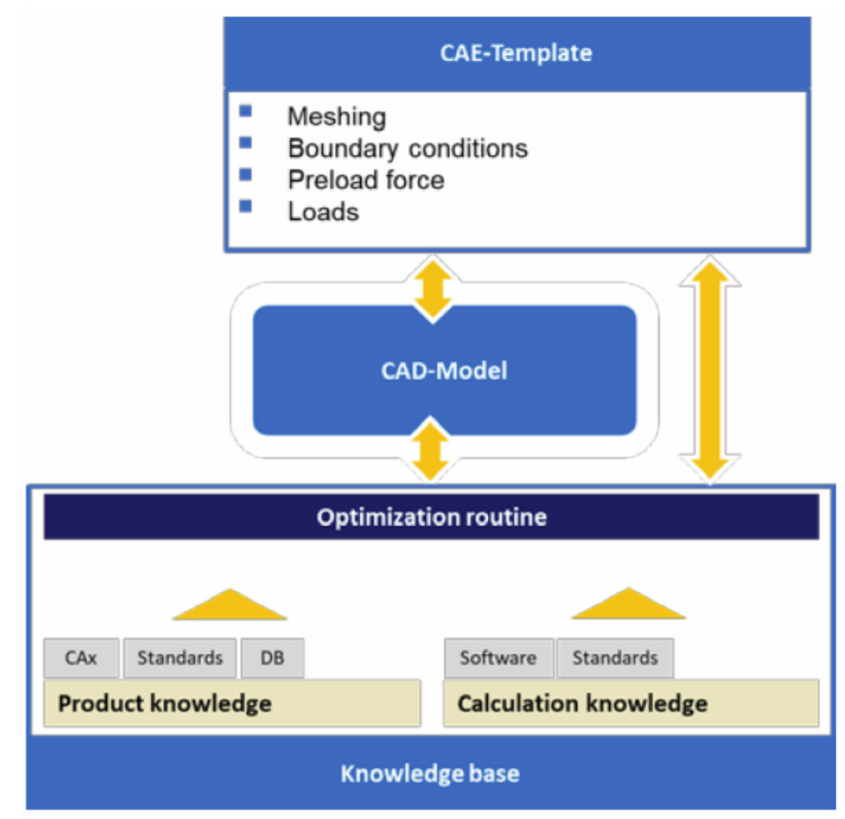

Figure 3. Procedure to optimize a component

In VDI 2230 Part 2 (VDI, 2014) alone, a calculation of screws using the Finite Element Method (FEM) is described. The remaining regulations are limited to the analytical calculation of screws and bolts. The contribution of FEM in designing the bolted joint depends primarily on modelling. In order to examine bolted connections in the FEM, four different model classes are described in the literature (compare Figure 4). With increasing model class, the degree of modelling and, thus, the significance of the calculation increases. Model class 3 has proven to be sufficient for calculating the flange connection. In model class 3, the screw is represented as a solid body. However, modelling is performed without displaying the thread; the effort needed for modelling this screw falls in the middle region. In order to simulate the real properties of a screw, the geometry or the material law can be adapted. The flexibility of the screw can be taken from tests or from VDI 2230 Part 1 (VDI, 2015). The contact in the parting line as well as in the contact surfaces can be taken into account (VDI, 2015).

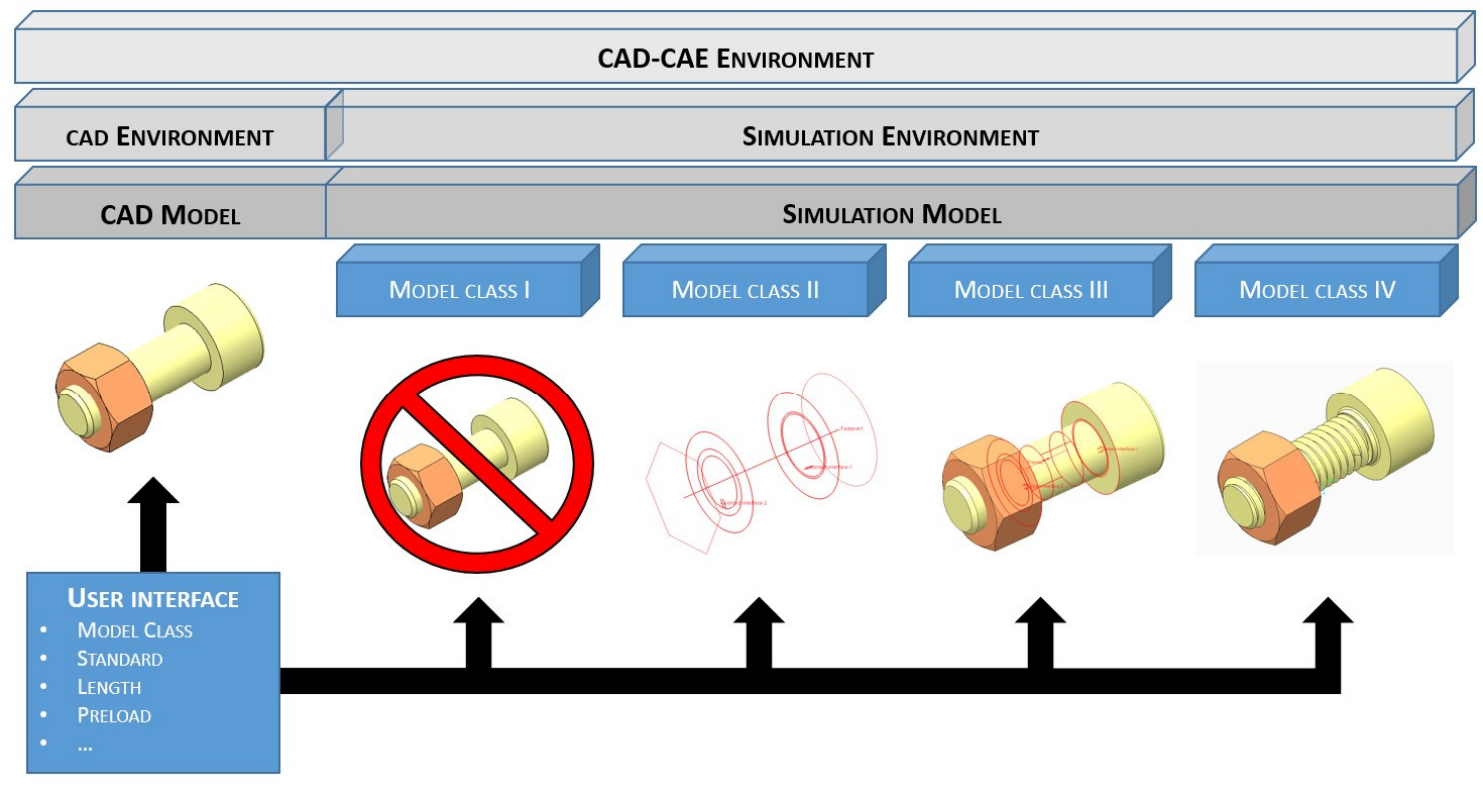

Figure 4. Bolt modell classes regarding VDI 2230 Part 2 (VDI, 2014) 
By considering the presented standards as well as the aforementioned demands on today's designer, it is clear that the current state of the art does not sufficiently support the designer. Regarding the simulation, which by no means is trivial, it is necessary to provide the designer with a tool that guides him or her through the process and, at best, covers all standard cases, thus enabling an automated optimization of the bolted joint as far as possible.

To accomplish this, a process needs to be developed that enables intelligent screw design. The previous optimization process of the bolt connection begins with the input of the loads such as temperature, bolt forces, internal pressure and external forces by the user. First, the thermal analysis is carried out followed by a coupling with the static-mechanical analysis. The respective results are transferred to the KissSoft ${ }^{\mathbb{B}}$ analysis software, where the safety of the components is calculated, see Figure 5 . The calculated safety factors are fed back to the simulation result and displayed there. The initial values for the simulation are not based on any analytical assumptions, but rather are empirically determined by the user. The user also interprets the results; he or she also has to assess the safety margins and decide whether they suffice. If the user concludes that they are insufficient, he or she intervenes in the process and controls it manually. Possible measures are to increase the screw strength class, replace the flange material or increase the bolt preload forces. The taken measures also depend on the respective user's experience and "gut feeling"; in other words, the measures have not been verified analytically prior to the new simulation. This lack of analytical verification leads to many iteration loops that, in turn, are time consuming and drive up costs.

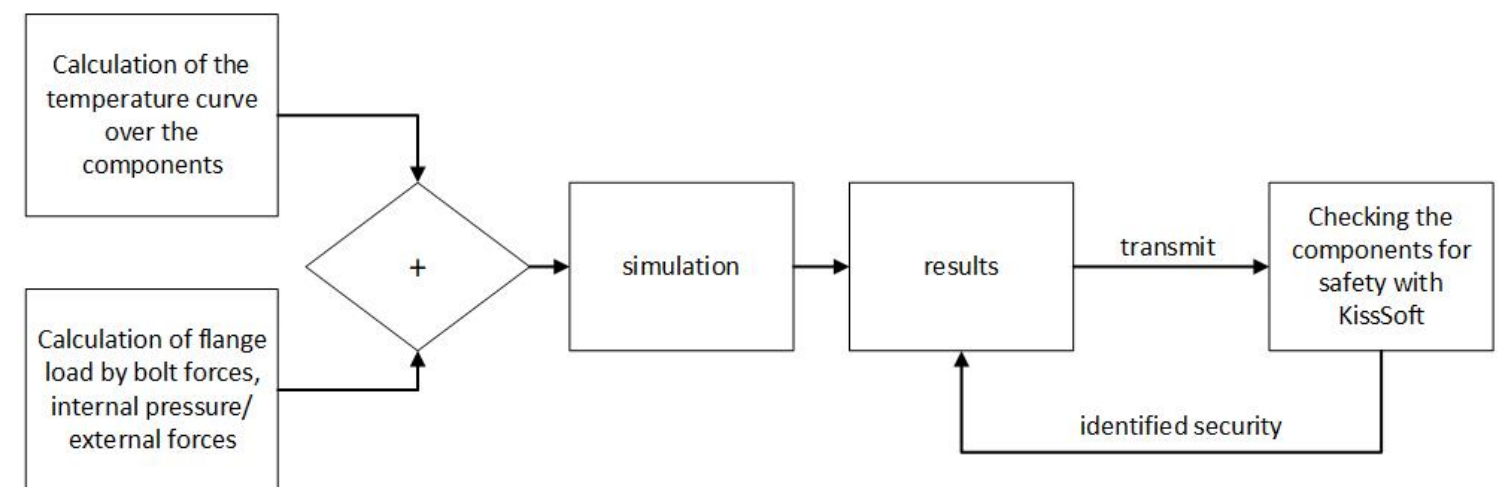

Figure 5. Diagram of optimization process according to flangeValid ${ }^{\circledR}$

Intelligent screw design needs to support the user beyond pre-processing, processing and postprocessing. Ideally, the user enters the boundary conditions, load factors and environmental parameters of the bolted joint into the simulation, and the KBE software performs all further steps. Figure 6 graphically illustrates such an optimization process. In the CAD environment, the user designs an assembly. The KBE software can be employed already here to support the user, because components are suggested and screws are selected by querying boundary conditions, field of application and loads. However, a prerequisite for such KBE software is that the corresponding knowledge can be stored, e.g. by applying standards or VDI guidelines.

If it is not possible to first use such KBE software, the KBE software is employed when switching to the CAE environment. The first step starts with the query of the occurring loads that are specified by the user.

During pre-processing, these parameters are stored in one or more spreadsheets where the bolt connection is predesigned. For example, calculation specifications are partly given in standards for special areas of mechanical engineering, e.g. for flange connections, in order to determine the necessary bolt force required to meet application-specific requirements, such as tightness. By coupling these values with VDI 2230 Part 1 (VDI, 2015), the screw safety can also be checked directly. The parameters thus obtained and verified for the bolt connection are fed back to the CAE environment and used as input values for the bolt preload force. The required material parameters and component sizes are retrieved from a database. 


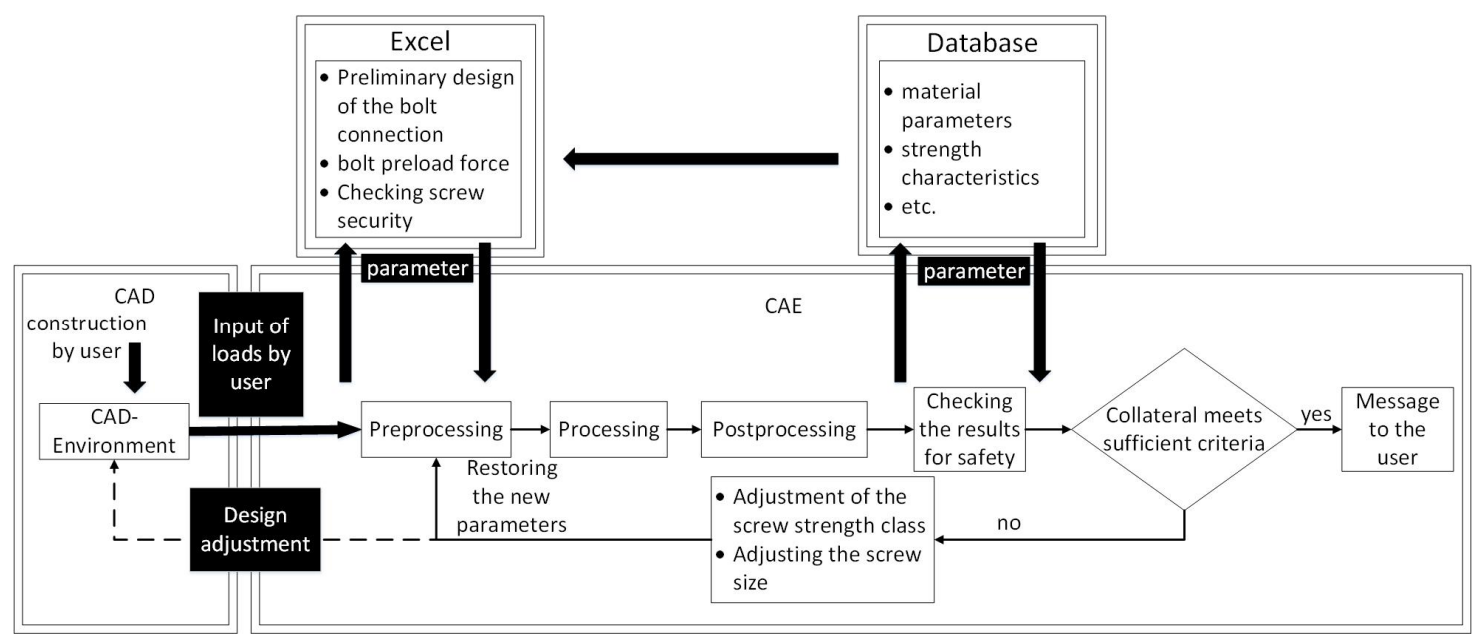

Figure 6. Model process of an optimization process for a bolted joint

The software also carries out further necessary pre-processing steps such as cross-linking, material allocation as well as the attachment of boundary conditions, forces and moments. In addition, the software figures the settings for the solver.

In pre-processing, the basics for post-processing are laid by positioning measuring points at already known, critical cross-sections. The CAE software then carries out the processing as usual. If the solver does not detect any errors in the simulation model, the user does not need to intervene.

Again, the KBE software executes the post-processing. The parameters stored in the measured values, such as contact pressure, reference voltage or displacement, are processed by the KBE software. For example, the results are compared using the characteristic values taken from the database, such as yield strength or screw strength, and the safety margins are determined. If the safety margins are sufficiently high, the user receives feedback from the KBE software about the successful dimensioning of the bolt connection. The simulation also produces a report containing the data relevant to the user. If the simulation results do not assure sufficient certainty, the optimization process begins. The respective optimization is carried out depending on which particular parameter target values have not been reached. For example, if the screw strength is not sufficient, the strength class of the screws is increased; if this does not suffice, the screw size is increased. Similarly, if a criterion such as tightness is not achieved, the bolt preload force is increased. The resulting values for these parameters are returned back to preprocessing and then replace the initial values of the calculation.

The coupling of CAE and CAD goes a step further here. Consider, for example, the simulation finding of insufficient safety for an M8 screw, even after the strength class had been increased. Moreover, consider that the use a M10 screw has been selected during the optimization process; subsequently, the 3D model will be adapted here. Consequently, the M8 screws will be replaced by M10 screws, and through holes and tapped holes will also be correspondingly adapted.

In both cases described above, the iteration loop is again repeated with pre-processing, processing, postprocessing and verification of the safety margins and the required criteria. This iteration process continues until the desired result has been achieved. The intelligent screw design presented here is now extended by an optimization. Here, the aim is to ensure that the sealing force on the flange seal is evenly distributed, even under unbalanced loads, see Figure 7.

One can discern four distinct areas: the area of design denoted by CAD, the area of simulation denoted by CAE, the database, and the evaluation routine. The optimization is started outside the areas. This is logical, because certain dimension and boundary conditions have to be defined before the design can begin. Subsequently, these boundary conditions and dimensions are entered into the database in the first step. Since the optimization routine aims to provide results for any flange connection, it is important that the design model, which is already stored in the CAD system, is a generic component or assembly. 


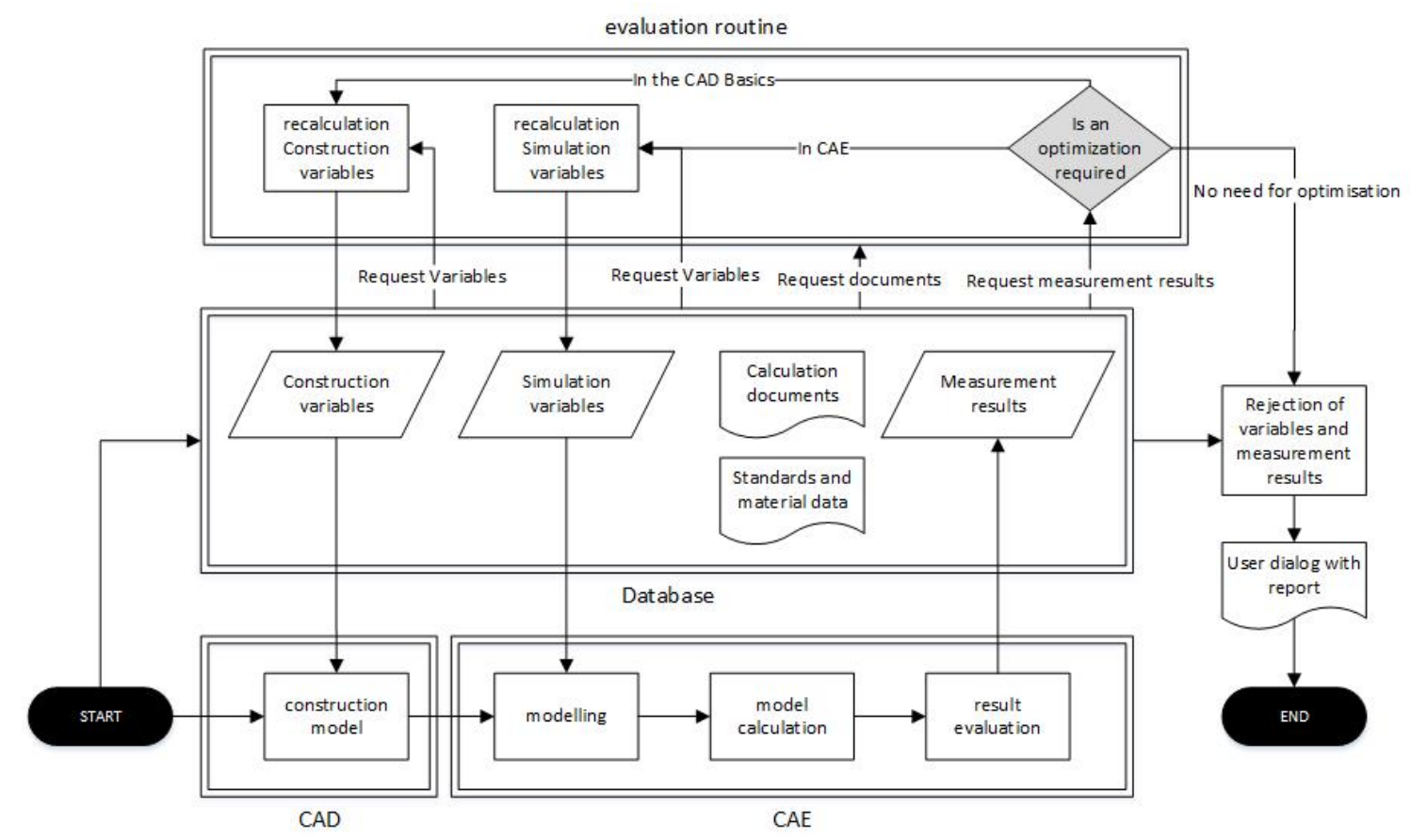

Figure 7. Optimization method

With the help of construction variables and parameters, an arbitrary flange connection is created from the generic assembly. When the data are entered into the database, all variables that completely describe a flange connection are therefore required. Since all elements of a flange connection are standard components, it is suitable to store the standards in the database. If all standards for the gasket, bolts and flanges have been defined, it is sufficient for the user to enter the following information:

- The flange type or its geometry code letter

- The pressure series

- The diameter series

- Additional specifications of the flange

- Characteristic values of the gasket used

- Characteristic values of the bolted joint used.

Embedding a standard considerably simplifies and accelerates this process, because the user only has to select the flange type; the database provides all required variables. Without the standard, numerous parameters would have to be entered at the beginning of the routine.

Input values are also required for modelling in the CAE area. The data include the externally acting forces. These are first calculated in a separate tool, such as Rohr2, and are read into the database at the beginning of the routine. Boundary conditions such as internal pressure and operating temperatures can also be read in at this time.

The documents and material data that are required for the optimization calculation, as well as the calculation of some simulation boundary conditions, need to be already stored in the database. This also applies to the aforementioned standards required for the derivation of the assembly. Importing the documents at the beginning of the routine would be another routine task that could slow down the process and cause errors.

After the flange assembly is built by means of design variables and after the external forces are imported, the newly created design assembly is transferred to the simulation environment. Modelling is also taken over by the routine. To be able to do this, it is necessary to automate the power generation, the formation of contact areas and the generation of loads and strains. The measurement variables for post-processing are also defined in this step. Even though the measurement types used are always the same, it should be 
possible to deselect certain measurement types. Therefore, the user is free to consider only individual measured variables during the optimization and to optimize the flange assembly accordingly.

Besides the changing of the number of screws, the geometry of the sealing surface can also change. This point needs to be considered upon automatically generating bolt pre-tensioning forces and cross-linking of the sealing surfaces. The program also needs to recognize how many bolt connections the flange connection contains and which areas are provided with a mesh refinement in order to achieve sufficient accurate results.

The pre-tensioning forces of the bolt connection have already been calculated once in this step. This calculation is carried out directly when the external forces are imported into the database, so that the starting preload forces can be transferred from the database into the simulation environment. The calculation is based on DIN EN 1591-1 (DIN, 2013). Up to this step and stemming from a generic assembly, a specific assembly was derived for which a calculation using pre-processing was prepared. The routine now has to start the model calculation. The settings of the solution solver should also be taken over by the routine and adjusted by the routine if there is an erroneous calculation run.

\section{Implementation}

The two methods presented above have already been implemented using an application example. For this purpose, the automated simulation model was first built in Siemens NX10. Furthermore, the developed method can be implemented in any other Product Lifecycle Management (PLM) system with an Application Programming Interface (API).

As shown in Figure 8, the user is guided through the simulation process and finds a Graphical User Interface (GUI) interface where he/she can enter the relevant information for the calculation. The simulation model is then built up automatically, whereby the specified loads and an approximate bolt preload force calculated according to DIN EN 1591-1 (DIN, 2013) are applied. After simulation, the results are compared with the strength values of the bolts and gasket. If a limit value is exceeded or falls short, the preload force is adjusted accordingly or the existing bolts are recommended to be replaced by a higher nominal size.

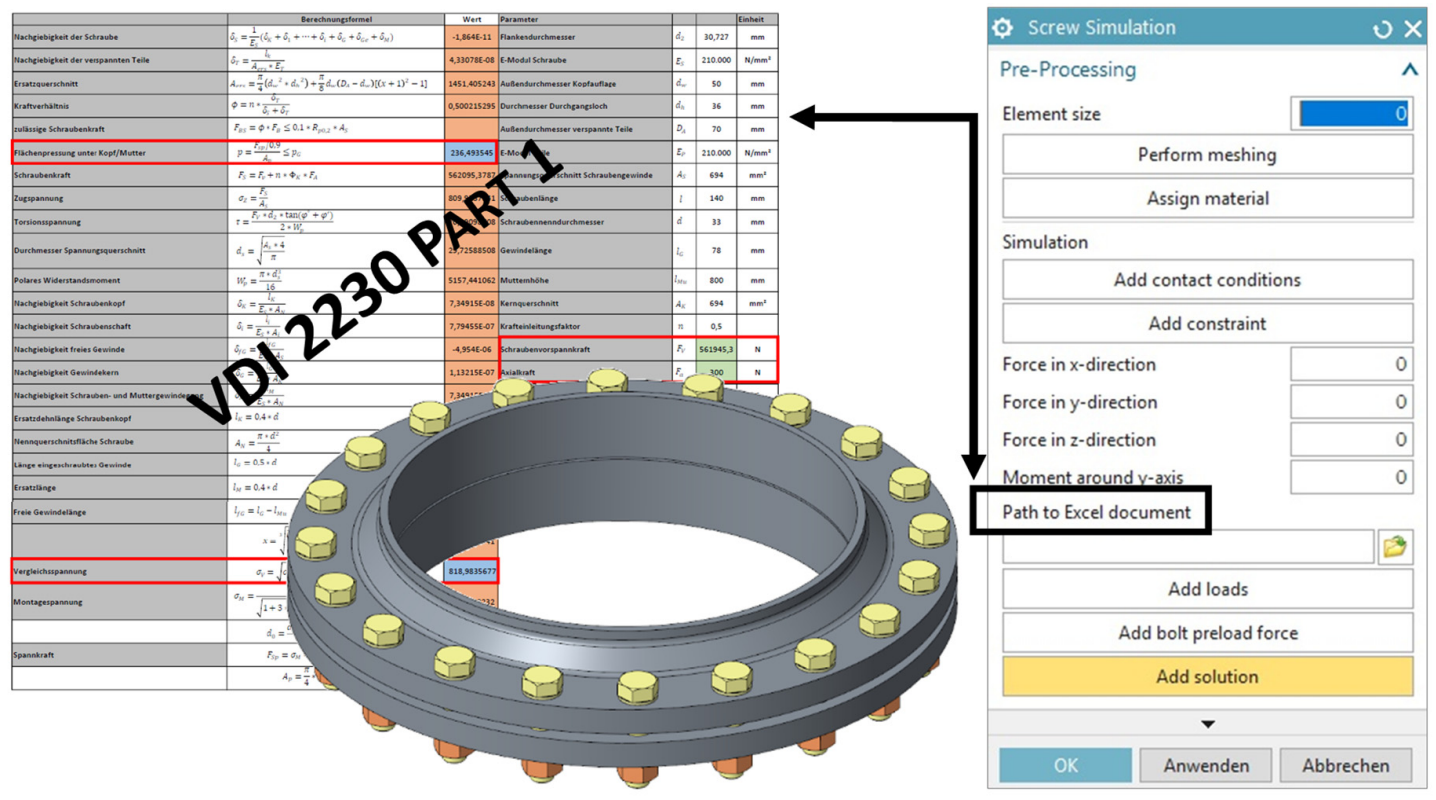

Figure 8. Screw simulation

Basically, the integration of the approach for optimizing a bolted joint can be evaluated positively. The linking of Siemens NX10 and Excel takes place without major problems and the exporting of 
measurement results into tables and the traceability of calculated values to Siemens NX10 works smoothly. Problems and disadvantages of this method will be discussed in the section "Discussion". While the optimization was implemented, it was recognized that the axes of a flange cannot be used for its alignment. Since the axes have no direct relation to the flange-sealing surface, they have no influence on the pressure distribution established on this surface. They could only be used for theoretically describing the position relation of flanges; yet they were not useful for optimization during simulation. Too many factors can influence the results when using axes. Instead of the axes, distance measurements between the bolt head and the nut of each bolt connection were used as a solution for the task. Compared to the axes, these are much more precise in determining the flange surface alignment.

Consequently, it was decided to develop a new approach for simulation-accompanying optimization. This approach was implemented by means of pressure measurements on the gasket. The measuring points for the pressure measurements were distributed evenly on a circle which was located near the highest surface pressure. Upon evaluating the measurements, it was found that the number of measuring points was distributed evenly over the number of screws. For the number of 40 measuring points selected here, ten measuring points were assigned to each of the four screws of the selected example flange assembly: five on one side and five on the other. Thus, each screw was assigned a range of 90 degrees of pressure measurement. The mean value was determined from this measuring range and compared with the other mean values of the other screws. The bolt preload force of the screw with the lowest mean value was increased by a certain amount according to a calculation rule. This amount changed as the optimization progressed, so that the optimization was able to achieve a better resolution towards the end.

By using this method, it was possible to adjust the contact pressure between the gasket and the flange much better. The deviation of the mean value of the pressure measurement of the individual ranges could be improved from about $30 \mathrm{MPa}$ to about $1.5 \mathrm{MPa}$. This corresponds to an optimization of $95 \%$ which, at the same time, corresponded to the set optimization criterion, see Figure 9. If a different criterion had been chosen, the value could have been further optimized. However, noteworthy here is the fact that with more precise results, the steps of optimization become increasingly finer; thus, the number of necessary optimization loops does not increase linearly, but rather exponentially.

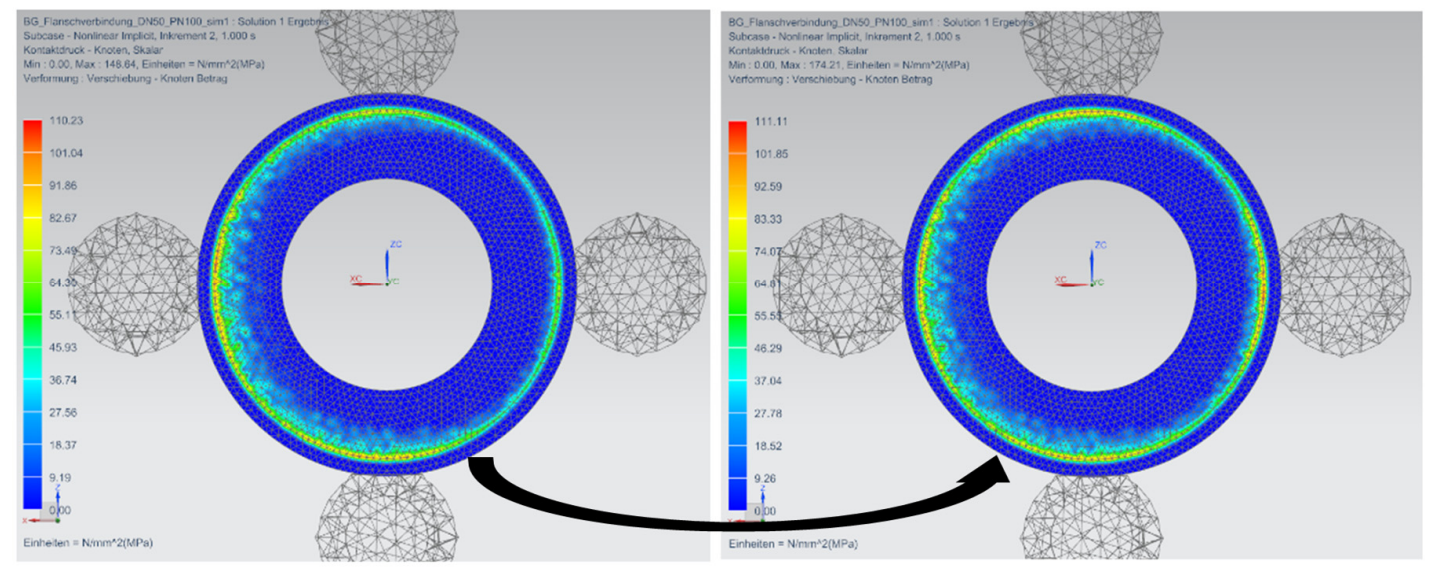

Figure 9. Optimization of contact pressure

In this paper, the theoretical basics dealt with the creation of a flange connection. Common methods were explained which allow the same pretensioning forces to be adjusted uniformly on all bolted connections of the flange connection. Now the pretensioning forces of the example assembly were changed so that the contact pressure is optimized, but the pretensioning forces are all different from each other.

The potential of coupling the methods presented here is demonstrated by the fact that it is possible to automatically calculate flange preload forces. Suitable templates and parameters have to be defined for the optimization. Even though developing them can take considerable time, this is beneficial for optimization calculations such as flanges or components with similar properties. 


\section{Discussion}

The methods for creating and optimizing simulation models presented in this work could be applied to the example and implemented. Hereby, it was shown that a two-stage implementation is advantageous. Problems in the implementation of the simulation model could be solved during the optimization process. Nevertheless, some questions still remain unanswered. For example, it has to be questioned how practical it is to tighten bolts with different torques in a flange connection. For this purpose, numerous aspects need to be taken into account, including the installation direction and orientation of the flange, which is determined by this procedure. Even so, both methods can be applied to other problems and support interdisciplinary engineers in their work. These methods represent a further step towards designing components during construction, which will inherently bolster cost-effectiveness, ultimately leading to cost savings during construction. This approach will not replace calculation engineers but rather will only shift their activity.

\section{Outlook and summary}

In this article it was shown how simulation models, based on the example of flange connections, can be built up knowledge-based, so that they can be carried out mostly automatically. In a further step, a method for optimizing the flange connection was presented. Parameters were identified that are suitable for optimization. The next steps are to present the ideas to industry partners in order to receive feedback. It is also conceivable to transfer the developed methods into a micro-service and thus make them available independently of the platform and to store the integrated knowledge in a central knowledge base.

\section{References}

ASME (2013), BPVC Section VIII - Rules for Construction of Pressure Vessels, ASME, New York.

Boussuge, F., Shahwan, A., Leon J.-C., Hahmann S., Foucault G. and Fine L. (2014), “Template-based Geometric Transformation of a Funcionally Enriched DMU into FE Assembly Models", Computer-Aided Design and Applications, Vol. 11 No. 4, pp. 436-449. https://doi.org/10.1080/16864360.2014.881187

Danjou, S., Lupa, N. and Köhler, P. (2008), “Approach for Automated Product Modeling Using Knowledge-Based Design Features", Computer-Aided Design and Applications, Vol. 5 No. 5, pp. 622-629. https://doi.org/10.3722/cadaps.2008.622-629

Deng, Y.-M., Britton G.A., Lam, Y.C., Tor, S.B. and Ma, Y.S. (2002), "Feature-based CAD-CAE integration model for injection-moulded product design", International Journal of Production Research, Vol. 40 No. 15, pp. 3737-3750. https://doi.org/10.1080/00207540210141643

DIN (2013), EN 1591-1 Flanges and their joints - Design rules for gasketed circular flange connections - Part 1: Calculation, German version EN 1591-1:2013, Beuth Verlag, Berlin.

DIN (2016), AD2000-Merkblatt B7:2016-09 Boltings, Beuth Verlag, Berlin.

DIN (2017), AD2000-Merkblatt B8:2017-06 Flanges, DIN, Berlin.

Hagenrainer, T. (2016), Ansätze zur Effizienzsteigerung und impliziten Wissensvermittlung in der Konzeptphase der virtuellen Entwicklung von Serienprodukten, $\mathrm{PhD}$ thesis, University of Duisburg-Essen, Duisburg.

Humpa, M. and Köhler, P. (2014), "Design Approach for Spiral Milling Parts Using Knowledge Based Engineering", Proceedings of 27th International Conference on CAD / CAM: Robotics of the Future 2014, London, UK, July 22-24, 2014, IOP Publishing, Bristol. https://doi.org/10.1088/1757-899X/65/1/012001

Kesselmans, C. (2014), Höherwertige Konstruktionsobjekte für CAD-Prozesse, PhD thesis, University of Duisburg-Essen, Duisburg.

Kestel, P. and Wartzack, S. (2016), "Wissensbasierter Aufbau konstruktions-begleitender Finite-ElementeAnalysen durch ein FEA-Assistenzsystem”, Entwickeln - Entwerfen - Erleben 2016 - Beiträge zur virtuellen Produktentwicklung und Konstruktionstechnik (EEE'2016), Dresden, Germany, June 30 - July 1, 2016, TUDpress, Dresden, pp. 315-329.

Klemme, U. (2015), Methoden zum wissensbasierten Aufbau simulationsorientierter Produktmodelle, $\mathrm{PhD}$ thesis, Universität Duisburg-Essen.

Lee, S.H. (2005), "A CAD-CAE integration approach using feature-based multi-resolution and multi-abstraction modelling techniques", Computer-Aided Design, Vol. 37 No. 9, pp. 941-555. https://doi.org/10.1016/j.cad.2004.09.021 
Manoharan, T., Humpa, M., Martha, A. and Köhler, P. (2016), "Knowledge integration in CAD-CAM process chain", Computer-Aided Design and Applications, Vol. 13 No. 5, pp. 729-736. https://doi.org/10.1080/16864360.2016.1150720

Roller, D. (1995), CAD Effiziente Anpassungs- und Variantenkonstruktion, Springer, Berlin, Heidelberg. https://doi.org/10.1007/978-3-642-79449-0

Szeghö, K. and Bercsey, T. (2007) "Kosten- und Risikomanagement in der frühen Phase der Produktentwicklung", Proceedings of the 18th Symposium on Design for X (DFX 2007), Neukirchen/Erlangen, Germany, October 11-12, 2007, pp. 65-72.

VDI (2003), VDI 2211 Information technology in product development - Calculation in design, Beuth Verlag, Berlin.

VDI (2014), VDI 2230 Part 2 Systematic calculation of highly stressed bolted joints, Beuth Verlag, Berlin.

VDI (2015), VDI 2230 Part 1 Bolt calculation, Beuth Verlag, Berlin.

VDI (2017), VDI 5610 Part 2 Knowledge management for engineering - Knowledge based engineering (KBE), Beuth Verlag, Berlin.

André Loibl, Research Associate

Universität Duisburg-Essen, Rechnereinsatz in der Konstruktion

Lotharstraße 1, 47057 Duisburg, Germany

Email: andre.loibl@uni-due.de 